\title{
O processo de enfrentamento da pandemia de COVID-19 na perspectiva de
}

\section{profissionais da Enfermagem}

\author{
The process of coping with the COVID-19 pandemic from the perspective of nursing professionals \\ El proceso de afrontamiento de la pandemia de COVID-19 desde la perspectiva de los profesionales \\ de enfermería
}

Recebido: 16/12/2020 | Revisado: 24/12/2020 | Aceito: 29/12/2020 | Publicado: 03/01/2021

Célia Maria Gomes Labegalini

ORCID: https://orcid.org/0000-0001-9469-4872

Universidade Estadual do Paraná, Brasil

E-mail: celia.labegalini@gmail.com

Kely Paviani Stevanato

ORCID: https://orcid.org/0000-0003-1872-8246

Universidade Estadual do Paraná, Brasil

E-mail: kelypaviani@hotmail.com

Iara Sescon Nogueira

ORCID: https://orcid.org/0000-0001-5815-9493

Universidade Estadual do Paraná, Brasil

E-mail: iarasescon@hotmail.com

Heloá Costa Borim Christinelli

ORCID: https://orcid.org/0000-0003-0772-4194

Universidade Estadual do Paraná, Brasil

E-mail: heloa.borim@hotmail.com

Vinícius Luís da Silva

ORCID: https://orcid.org/0000-0001-6228-8124

Universidade Estadual do Paraná, Brasil

E-mail: vinicius.luissilva@hotmail.com

Maria Antonia Ramos Costa

ORCID: https://orcid.org/0000-0001-6906-5396

Universidade Estadual do Paraná, Brasil

E-mail: maria.costa@unespar.edu.br

\begin{abstract}
Resumo
Atualmente o mundo vivencia uma pandemia causada pelo vírus SARS-CoV-2, o qual obrigou os serviços de saúde a se organizarem, sendo os gestores municipais de saúde, junto com as regionais de saúde os responsáveis por operacionalizar as ações preventivas no âmbito do município e a equipe de enfermagem responsável por realizar as ações. Objetivou-se compreender o processo de enfretamento da pandemia de COVID-19 na perspectiva de profissionais da enfermagem e de gestores municipais de saúde. Trata-se uma pesquisa qualitativa, descritiva e exploratória, realizada com 17 profissionais da enfermagem, por meio de um roteiro de questões. Os dados foram tabulados, organizados utilizando o software IRaMuTeQ ${ }^{\circledR}$ e analisados. Os participantes eram do sexo feminino, adultos jovens, sendo 12 enfermeiros e cinco técnicos de enfermagem. Exercem atividades de assistência e de coordenação, e seis exercem as duas atividades de forma concomitante, e atuam em diferentes níveis de atenção em saúde. O estudo apreendeu os sentimentos e o impacto na vida pessoal dos profissionais de Enfermagem por estarem na linha de frente da COVID-19, os desafios no enfrentamento da doença, bem como as lições advindas desse processo, as transformações no processo de trabalho e o papel dos profissionais de Enfermagem. A COVID-19 alterou os processos na saúde e a vida dos profissionais da Enfermagem.
\end{abstract}

Palavras-chave: Administração de serviços de saúde; Organização e administração; Enfermagem; Pandemias; Coronavírus; COVID-19.

\begin{abstract}
Currently the world is experiencing a pandemic caused by the SARS-CoV-2 virus, which forced health services to organize themselves, with municipal health managers, together with regional health managers, being responsible for implementing preventive actions within the scope municipality and the nursing team responsible for carrying out actions. The objective was to understand the process of coping with the COVID-19 pandemic from the perspective of nursing professionals and municipal health managers. It is a qualitative, descriptive and exploratory research, carried out with 17 nursing professionals, through a script of questions. The data were tabulated, organized using the IRaMuTeQ ${ }^{\circledR}$ software and combats. Participants were female, young adults, 12 nurses and five nursing technicians. They perform assistance and coordination activities, and six exercises as two activities concomitantly, and operate at
\end{abstract}


different levels of health care. The study apprehended the feelings and the impact on the personal life of Nursing professionals for being on the front line of COVID-19, the challenges in coping with the disease, as well as the lessons from this process, the transformations in the work process and the role of nursing professionals. COVID-19 changed the processes in health and the lives of nursing professionals.

Keywords: Health services administration; Organization and administration; Nursing; Pandemics; Coronavirus; COVID-19.

\section{Resumen}

Actualmente el mundo vive una pandemia provocada por el virus SARS-CoV-2, que obligó a los servicios de salud a organizarse, siendo los gerentes de salud municipales, junto con los gerentes regionales de salud, los encargados de implementar acciones preventivas dentro del municipio y el equipo de enfermería responsable de realizar las acciones. El objetivo fue comprender el proceso de afrontamiento de la pandemia de COVID-19 desde la perspectiva de los profesionales de enfermería y los gestores de salud municipales. Se trata de una investigación cualitativa, descriptiva y exploratoria, realizada con 17 profesionales de enfermería, a través de un guión de preguntas. Los datos se tabularon, organizaron con el software IRaMuTeQ ${ }^{\circledR}$ y se analizaron. Los participantes fueron mujeres, adultos jóvenes, 12 enfermeras y cinco técnicos de enfermería. Realizan actividades de asistencia y coordinación, y seis realizan ambas actividades simultáneamente y operan en diferentes niveles de atención de salud. El estudio aprehendió los sentimientos y el impacto en la vida personal de los profesionales de Enfermería por estar en la primera línea del COVID-19, los desafíos en el afrontamiento de la enfermedad, así como las lecciones de este proceso, las transformaciones en el proceso de trabajo y el rol de los profesionales de enfermería. COVID-19 cambió los procesos en salud y la vida de los profesionales de enfermería.

Palabras clave: Administración de los servicios de salud; Organización y administración; Enfermería; Pandemias; Coronavirus; COVID-19.

\section{Introdução}

Atualmente, no ano de 2020, o mundo vivencia uma pandemia ocasionada pelo novo coronavírus, o vírus SARS-CoV2, causador da doença denominada COVID-19. É uma doença nova que atinge o sistema respiratório e foi identificada pela primeira vez em Wuhan, na China, em dezembro de 2019 (Brasil, 2020a). Sua transmissão ocorre a partir da disseminação do vírus de uma pessoa doente para outra, ou por contato próximo por meio do aperto de mão, gotículas de saliva, espirro, tosse, catarro, objetos e/ou superfícies contaminadas, tendo como sintomas a febre, tosse ou dificuldade para respirar, entre outros sintomas gripais (Brasil, 2020d).

A Organização Mundial de Saúde (OMS) declarou, em 30 de janeiro de 2020, que se trata de uma emergência de saúde pública de importância internacional devido ao alto número de pessoas infectadas e óbitos por essa doença. O Ministério da Saúde (MS) declarou em março de 2020 que em todo o território nacional há transmissão comunitária da doença (Brasil, 2020a). Até a data de 13 de dezembro de 2020, foram confirmados no Brasil, 6.901.952 casos da COVID-19 e 181.402 mortes, com taxa de mortalidade de 86,3 a cada 100 mil habitantes. No estado do Paraná, até a mesma data, registraram 329.782 casos e 6.764 óbitos, com taxa de mortalidade 59,2, cerca de 31\% menor que a média nacional (Brasil, 2020b).

Um estudo publicado na revista Nature revelou que a doença COVID-19 está se tornando imparável, e portanto, necessita de uma resposta global coordenada e que seja necessária para preparar os sistemas de saúde para o enfrentamento da doença (Callaway, 2020). Diante deste cenário, os países estão organizando ações em saúde para enfrentar a doença. No Brasil, o MS publicou em 13 de fevereiro de 2020 o Plano de Contingência Nacional para Infecção Humana pelo novo coronavírus COVID-19, formulado pelo Centro de Operações de Emergências em Saúde Pública (COE-COVID-19) (Brasil, 2020c).

A partir desse momento, uma série de ações foram adotadas, culminando com a ativação, no dia 22 de janeiro de 2020 , do Centro de Operações de Emergência em Saúde Pública (COE-COVID-19) do MS, coordenado pela Secretaria de Vigilância em Saúde (SVS), com o objetivo de nortear a atuação do MS na resposta à possível emergência de saúde pública, buscando uma atuação coordenada no âmbito do SUS (Brasil, 2020c).

Assim, para o enfrentamento da pandemia da COVID-19, os serviços de saúde precisaram se reorganizar, sendo que os gestores municipais de saúde, juntamente com as regionais de saúde, foram os responsáveis por operacionalizar as ações 
preventivas no âmbito do município e também de estruturar os serviços e capacitar os profissionais envolvidos com a linha de frente. Ainda, para que essas ações fossem efetivas, tornou-se necessário a articulação com demais setores da sociedade (Araújo, Oliveira \& Freitas, 2020; Daumas et al., 2020).

A rápida disseminação do vírus no mundo exigiu medidas emergenciais e muitas vezes sem o norteamento padronizado, e dessa forma, coube aos gestores municipais a iniciativa no processo decisório local. Além disso, a pandemia exigiu capacitação dos profissionais, especialmente da enfermagem, para o enfretamento de uma doença respiratória com transmissão por gotícula, a qual necessita de uso de equipamentos de proteção individual, os quais não fazem parte da rotina diária de muitos profissionais de saúde (Araújo, Oliveira \& Freitas, 2020; Daumas et al., 2020; Helioterio et al., 2020; Lana et al., 2020).

Ainda, a virulência e letalidade da COVID-19 impactou o processo de trabalho dos profissionais de saúde, bem como o manejo da doença dos diversos níveis de assistência, carecendo de preparo técnico. Cabe destacar, a enfermagem como categoria profissional que presta o cuidado direto e contínuo ao paciente, e seu papel primordial no acesso a saúde e na manutenção da vida, especialmente durante a pandemia (Araújo, Oliveira \& Freitas, 2020; Daumas et al., 2020; Helioterio et al., 2020; Lana et al., 2020).

Conhecer o processo de organização dos serviços pelos gestores, destacando as ações realizadas, os setores envolvidos e o apoio recebido diante do processo decisório, bem como a perspectiva dos profissionais de enfermagem envolvidos na linha de frente do cuidado, possibilita compreender os fatores de apoio e os desafios que permeiam esse processo. Tais dados podem subsidiar novas práticas e gerar informações que auxiliem no preparo e atuação dos profissionais de saúde no enfretamento da pandemia de COVID-10, que ainda encontra-se latente, e ocasionalmente de novas pandemias que poderão vir a surgir.

Dessa forma, o estudo assumiu a seguinte pergunta de pesquisa: Como está sendo realizado o enfrentamento da pandemia da COVID-19, pelos profissionais da enfermagem e gestores municipais? O objetivo portanto, foi compreender o processo de enfretamento da pandemia de COVID-19 na perspectiva de profissionais da enfermagem e de gestores municipais de saúde.

\section{Metodologia}

Tratou-se uma pesquisa qualitativa, descritiva e exploratória, desenvolvida com 17 profissionais de enfermagem que possuem proximidade com a gestão e/ou assistência ao COVID-19, atuantes em um município de médio porte, localizado no Noroeste do estado do Paraná, Brasil. Os critérios de inclusão foram: ser profissional da enfermagem, atuar a mais de um ano na referida instituição, e estar atuando na gestão ou assistência ao COVID-19. Critérios de exclusão: Não estar exercendo atividades laborais devido férias, licença ou atestado durante o período de coleta de dados do estudo.

A coleta de dados foi realizada durante os meses de junho a julho de 2020, por meio remoto, utilizando um formulário on-line elaborado na plataforma Google®. O mesmo foi elaborado pelos pesquisadores e foi composto por questões de caracterização sociodemográfica e outras que versavam sobre a temática, destinados aos gestores e aos profissionais da enfermagem. O convite para participar do estudo bem como o Termo de Consentimento Livre e Esclarecido foi enviado para os participantes por e-mail e por aplicativo de mensagem de texto, nos endereços eletrônicos fornecidos pela secretaria municipal de saúde. Foi solicitado que os participantes encaminhassem aos seus pares, utilizando assim a técnica "bola-de-neve", até que houve a saturação das respostas.

Os dados obtidos nas entrevistas foram processados e organizados utilizando o software IRaMuTeQ®, a partir da Classificação Hierárquica Descendente. O processamento do corpus proveniente das entrevistas com os profissionais de saúde identificou 3.179 ocorrências de palavras, distribuídas em 603 formas ativas e divididas em 65 Unidades de Contexto Elementar (UCEs), com aproveitamento de 75,58\% do corpus. O tempo de processamento pelo programa foi de 41 segundos.

O estudo seguiu todos os preceitos éticos da Resolução 466/2012 e da Resolução 580/2018 do Conselho Nacional em 
Saúde, sendo aprovada pelo Comitê de Ética em Pesquisa da Universidade Estadual do Paraná sob número 4.014.513/2020 (CAAE: 30878720.9.0000.9247). Os participantes foram identificados com a letra 'P' seguindo de número arábico correspondente a ordem da transcrição, e de forma a preservar o seu anonimato.

\section{Resultados}

Os participantes foram predominantemente do sexo feminino ( $n=16)$, com idades de 29 a 45 anos e média de 37 anos. Em relação à categoria profissional, 12 eram enfermeiros e cinco técnicos de enfermagem. A maioria $(\mathrm{n}=10)$ atua na área a mais de 10 anos, cinco atuam entre 5 a 10 anos, e dois a menos de 5 anos. Os locais de atuação dos profissionais foram: sete no Serviço Móvel de Urgência, cinco em Unidades Básicas de Saúde (UBS), três em cargos de gestão/coordenação, dois em hospitais e um em uma Unidade de Pronto Atendimento (UPA).

Um dos participantes possuía dois vínculos empregatícios. Exercem atividades de assistência $(n=14)$ e de coordenação $(n=9)$, seis exercem as duas atividades de forma concomitante. Os que atuam nas áreas administrativas são vinculados a gestão da Atenção Primária à Saúde, na direção da Vigilância em Saúde, na Coordenação das UBS e na Central de Controle de Infecção Hospitalar.

Foram originadas do dendrograma da Classificação Hierárquica Descendente (CHD) seis classes de análise (Figura 1). A CHD dividiu as UCEs em duas repartições: da primeira repartição obteve-se as classes 1 e 5 (UCE = 30,8\%), e da outra repartição obteve-se a classe 6 (UCE=15,4\%), seguida de outras duas sub-repartições. De uma sub-repartição originou a classe $2(\mathrm{UCE}=16,9 \%)$, e da outra sub-repartição as classes 3 e $4(\mathrm{UCE}=37 \%)$.

Tais classes, interpretadas a partir dos enfrentamentos vivenciados pelos profissionais de enfermagem acerca da pandemia de COVID-19, estão apresentadas em formato de dendrograma (Figura 1) e em formato de quadro (Quadro 1). 
Figura 1 - Dendrograma de nuvem de palavras relacionadas ao enfrentamento dos profissionais de enfermagem acerca da pandemia de COVID-19, município do Noroeste do estado do Paraná, Brasil, 2020.

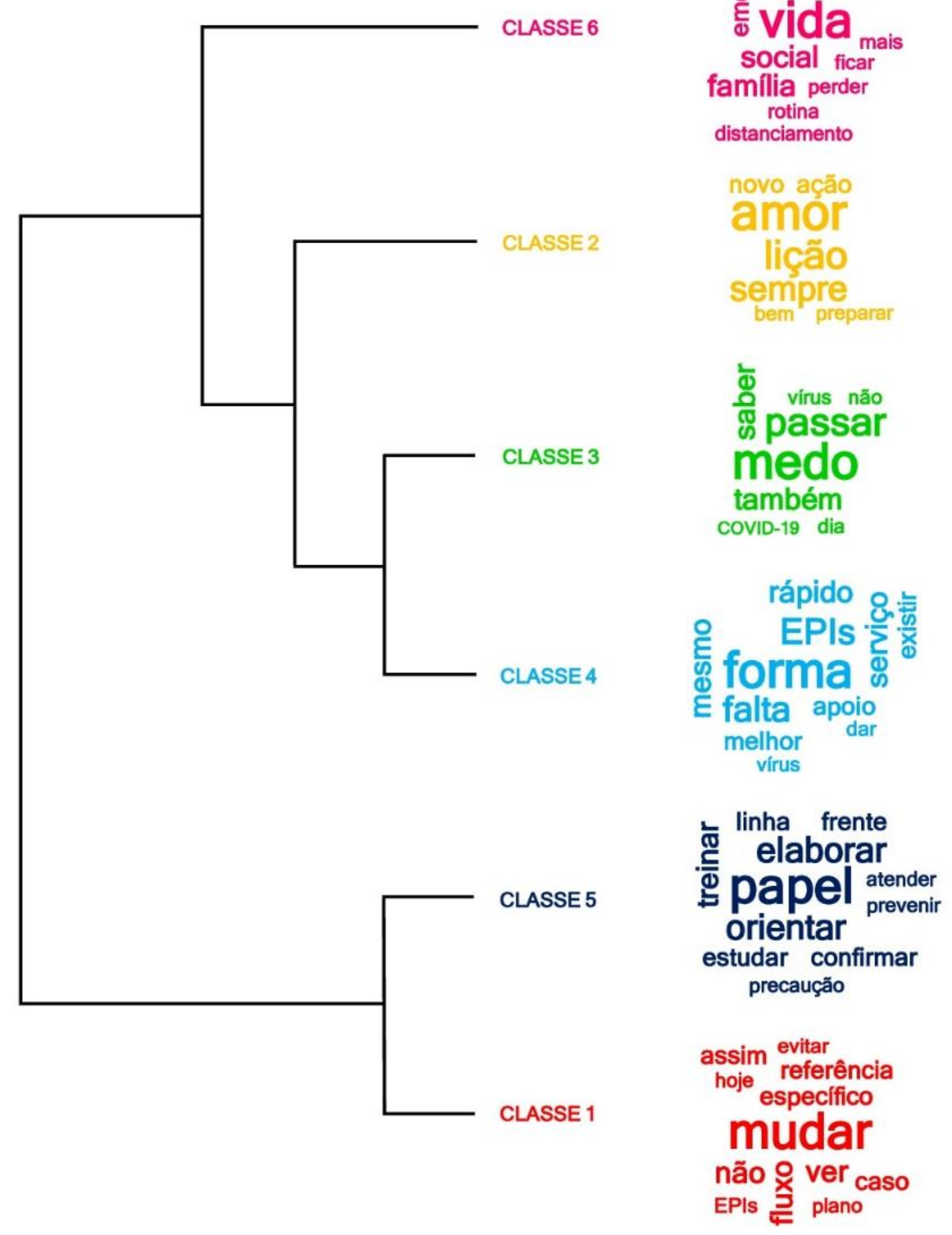

Fonte: Autores (2020). Organizado pelo software IRaMuTeQ®. 
Quadro 1 - Análise lexicográfica das classes referentes ao enfrentamento dos profissionais de enfermagem acerca da pandemia de COVID-19, listadas conforme dendrograma da CHD do corpus textual, município do Noroeste do estado do Paraná, Brasil, 2020 .

\begin{tabular}{|c|c|c|c|c|c|}
\hline \multirow[b]{2}{*}{ Classes } & \multirow[b]{2}{*}{ Nomenclatura } & \multicolumn{4}{|c|}{ Análise Lexicográfica } \\
\hline & & $\begin{array}{c}\text { Palavras } \\
(\mathrm{p}<0,05)^{*}\end{array}$ & $\begin{array}{c}\text { eff. } \\
\text { total } * *\end{array}$ & $X^{2 * * *}$ & $\% * * * *$ \\
\hline \multirow{10}{*}{$\begin{array}{c}\text { Classe } 3 \\
18,5 \% \\
12 \mathrm{UCE}\end{array}$} & \multirow{10}{*}{$\begin{array}{l}\text { Sentimentos negativos vivenciados } \\
\text { pelos profissionais de Enfermagem no } \\
\text { enfrentamento da COVID-19 }\end{array}$} & Descobrir & 5 & 13.63 & 80.0 \\
\hline & & Momento & 10 & 13.55 & 60.0 \\
\hline & & Medo & 13 & 13.52 & 53.85 \\
\hline & & Passar & 6 & 10.2 & 66.67 \\
\hline & & Difícil & 6 & 10.2 & 66.67 \\
\hline & & Contaminar & 5 & 6.21 & 60.0 \\
\hline & & Parar & 5 & 6.21 & 66.67 \\
\hline & & Insegurança & 3 & 4.86 & 66.67 \\
\hline & & Doença & 3 & 4.86 & 66.67 \\
\hline & & Cura & 3 & 4.86 & 66.67 \\
\hline \multirow{11}{*}{$\begin{array}{c}\text { Classe } 4 \\
18,5 \% \\
12 \mathrm{UCE}\end{array}$} & \multirow{11}{*}{$\begin{array}{l}\text { Desafios no enfrentamento da } \\
\text { COVID-19: aspectos psicológicos e } \\
\text { dificuldade na execução do cuidado }\end{array}$} & Desafio & 11 & 25.9 & 72.73 \\
\hline & & Forma & 5 & 13.63 & 80.0 \\
\hline & & Falta & 5 & 13.63 & 80.0 \\
\hline & & EPIs & 8 & 11.75 & 62.5 \\
\hline & & Rápido & 9 & 9.55 & 55.56 \\
\hline & & Melhor & 4 & 9.05 & 75.0 \\
\hline & & Apoio & 4 & 4.86 & 75.0 \\
\hline & & Psicológico & 3 & 4.86 & 66.67 \\
\hline & & Serviço & 3 & 4.86 & 66.67 \\
\hline & & Saúde & 3 & 4.86 & 66.67 \\
\hline & & Contaminação & 3 & 4.86 & 66.67 \\
\hline \multirow{9}{*}{$\begin{array}{c}\text { Classe } 2 \\
16,9 \% \\
11 \mathrm{UCE}\end{array}$} & \multirow{9}{*}{$\begin{array}{l}\text { Lições apreendidas pelos profissionais } \\
\text { de enfermagem durante o período da } \\
\text { pandemia de COVID-19: } \\
\text { transformação de saberes e práticas. }\end{array}$} & Lição & 8 & 21.89 & 75.0 \\
\hline & & Amor & 4 & 20.92 & 100.0 \\
\hline & & Enfermagem & 3 & 15.44 & 100.0 \\
\hline & & Sempre & 5 & 15.33 & 80.0 \\
\hline & & Precisar & 6 & 11.63 & 66.67 \\
\hline & & Próximo & 7 & 9.03 & 57.14 \\
\hline & & Mais & 12 & 6.41 & 41.67 \\
\hline & & Surgir & 3 & 5.54 & 66.67 \\
\hline & & Diário & 3 & 5.54 & 66.67 \\
\hline \multirow{13}{*}{$\begin{array}{c}\text { Classe } 6 \\
15,4 \% \\
10 \mathrm{UCE}\end{array}$} & \multirow{13}{*}{$\begin{array}{l}\text { Os impactos na vida pessoal em } \\
\text { relação aos aspectos emocionais, } \\
\text { familiares e sociais. }\end{array}$} & Impactar & 10 & 65.0 & 100.0 \\
\hline & & Pessoal & 10 & 65.0 & 100.0 \\
\hline & & Vida & 13 & 47.27 & 76.92 \\
\hline & & Social & 5 & 17.37 & 80.0 \\
\hline & & Aspecto & 3 & 17.3 & 100.0 \\
\hline & & Família & 7 & 10.51 & 57.14 \\
\hline & & Ficar & 5 & 8.28 & 60.0 \\
\hline & & Emocional & 5 & 8.28 & 60.0 \\
\hline & & Rotina & 3 & 6.35 & 66.67 \\
\hline & & Perder & 3 & 6.35 & 66.67 \\
\hline & & Dúvida & 3 & 6.35 & 66.67 \\
\hline & & Distanciamento & 3 & 6.35 & 66.67 \\
\hline & & Contato & 3 & 6.35 & 66.67 \\
\hline
\end{tabular}


Research, Society and Development, v. 10, n. 1, e5410111252, 2021

(CC BY 4.0) | ISSN 2525-3409 | DOI: http://dx.doi.org/10.33448/rsd-v10i1.11252

\begin{tabular}{|c|c|c|c|c|c|}
\hline & & Pandemia & 7 & 4.55 & 42.86 \\
\hline & & Familiar & 4 & 3.92 & 50.0 \\
\hline \multirow{21}{*}{$\begin{array}{c}\text { Classe } 1 \\
15,4 \% \\
10 \mathrm{UCE}\end{array}$} & \multirow{21}{*}{$\begin{array}{c}\text { Transformações no processo de } \\
\text { trabalho dos profissionais de } \\
\text { enfermagem. }\end{array}$} & Atendimento & 14 & 23.9 & 57.14 \\
\hline & & Unidade Básica & 7 & 18.93 & 71.43 \\
\hline & & Suspeito & 10 & 18.07 & 60.0 \\
\hline & & Mudar & 3 & 17.3 & 100.0 \\
\hline & & Treinamento & 5 & 8.28 & 66.67 \\
\hline & & Fluxo & 3 & 6.35 & 66.67 \\
\hline & & Específico & 3 & 6.35 & 66.67 \\
\hline & & Referência & 3 & 6.35 & 66.67 \\
\hline & & Plano & 3 & 6.35 & 66.67 \\
\hline & & Planejar & 3 & 6.35 & 66.67 \\
\hline & & Evitar & 3 & 6.35 & 66.67 \\
\hline & & Encaminhar & 3 & 6.35 & 40.0 \\
\hline & & COVID-19 & 10 & 5.5 & 33.33 \\
\hline & & Equipe & 15 & 4.83 & 33.33 \\
\hline & & Caso & 7 & 4.55 & 42.86 \\
\hline & & Orientação & 4 & 3.92 & 50.0 \\
\hline & & EPIs & 8 & 3.43 & 37.5 \\
\hline & & Realizar & 8 & 3.43 & 37.5 \\
\hline & & Trabalho & 18 & 2.94 & 27.78 \\
\hline & & Confirmar & 5 & 2.52 & 40.0 \\
\hline & & Paciente & 14 & 2.35 & 28.57 \\
\hline \multirow{14}{*}{$\begin{array}{c}\text { Classe 5 } \\
15,4 \% \\
10 \mathrm{UCE}\end{array}$} & \multirow{14}{*}{$\begin{array}{c}\text { O papel dos profissionais de } \\
\text { Enfermagem no enfrentamento da } \\
\text { COVID-19: execução do cuidado e } \\
\text { coordenação da equipe. }\end{array}$} & Papel & 11 & 58.02 & 90.91 \\
\hline & & Orientar & 6 & 23.44 & 83.33 \\
\hline & & Elaborar & 4 & 11.64 & 75.0 \\
\hline & & Frente & 5 & 8.28 & 60.0 \\
\hline & & Protocolo & 5 & 8.28 & 60.0 \\
\hline & & Linha & 5 & 8.28 & 60.0 \\
\hline & & Treinar & 3 & 6.35 & 66.67 \\
\hline & & Prevenir & 3 & 6.35 & 66.67 \\
\hline & & Estudar & 3 & 6.35 & 66.67 \\
\hline & & Precaução & 4 & 3.92 & 50.0 \\
\hline & & Cuidado & 5 & 2.52 & 40.0 \\
\hline & & Atender & 5 & 2.52 & 40.0 \\
\hline & & Necessário & 5 & 2.52 & 40.0 \\
\hline & & Paciente & 14 & 2.38 & 28.57 \\
\hline
\end{tabular}

*Nível de significância da associação da palavra com a classe de p<0,05.

**Número de segmentos de texto no corpus que contém determinada palavra.

**** $\mathrm{X}^{2}$ de associação da palavra com a classe.

****Percentagem da ocorrência da palavra nos segmentos de texto da classe.

Fonte: Autores (2020). Organizado pelo software IRaMuTeQ®.

A Classe 3 (UCE $=18,5 \%$ ) desvelou os sentimentos de medo, insegurança, dúvidas e incertezas vivenciados pelos profissionais de enfermagem durante a execução de suas atividades laborais no enfrentamento da pandemia de COVID-19, bem como o medo de disseminação do vírus. Segundo os mesmos, é um momento desafiador, inusitado e que requerem mudanças necessárias nas práticas de cuidado, como observado nas seguintes UCEs: 
Por ser algo novo, o enfrentamento está sendo receoso, muita insegurança e cheio de dúvidas. Divergências de opiniões também dificultam o processo de descoberta. (P8)

Estamos passando por um processo doloroso de reconstrução de ideias e costumes, desconstrução de alguns hábitos também. Foi um momento de muita união de conhecimentos e práticas, para desenhar e estruturar o trabalho no município. (P17)

Estamos passando por um momento desafiador pois a cada dia recebemos novas orientações para colocar em prática. As vezes surge o sentimento de medo por ser algo desconhecido, medo de contaminar quem amamos, entretanto, tenho crescido muito profissionalmente e acredito que vamos vencer este momento difícil. (P11)

Rezando todos os dias para não se contaminar e também disseminar o vírus sem saber. (P4)

A Classe $4(\mathrm{UCE}=18,5 \%)$ aponta os desafios e as dificuldades vivenciadas pelos profissionais de enfermagem. De acordo com os profissionais, os desafios relacionam-se com os aspectos psicológicos, como desgaste emocional, o medo da contaminação e do novo, bem como aqueles relacionados com a dificuldade do processo de trabalho, como a falta de Equipamento de Proteção Individual (EPIs), falta de protocolos, e a dificuldade em realizar atendimento de modo remoto. Seguem os depoimentos:

Um novo desafio, o qual envolve um desgaste emocional e tomada de decisões que envolve muitas pessoas. (P5)

O desafio é o apoio psicológico por parte da empresa aos trabalhadores e o medo da contaminação e de contaminar familiares. (P4)

O desafio é lidar com um vírus altamente perigoso que se quer conseguimos combater. (P4)

O desafio é o enfrentamento de algo pouco conhecido, sem muitos estudos, e sem protocolo de tratamento definido e eficaz para o tratamento deste vírus. É também a rápida disseminação do vírus, e a falta de EPIs necessários. (P9)

O desafio é que enquanto não existir a cura para a COVID-19, é fazer a população cumprir as precauções necessárias, e que percebam a importância do isolamento social neste momento em que vivemos. (P12)

O desafio é manter a saúde mental da equipe, realizar o trabalho de forma satisfatória mesmo realizando grande parte do atendimento por telefone ou internet. Sem contar o cansaço psicológico e a pressão de trabalhar todos os dias com vários EPIs que não são confortáveis e nos causam machucados. (P6)

No início fiquei apreensiva mas agora estou mais estável com a situação. Nos meus serviços existe carência de EPIs, mas estamos dando nosso melhor. (P2)

A Classe 2 (UCE $=16,9 \%)$ evidencia as lições apreendidas pelos profissionais de enfermagem durante o período da pandemia de COVID-19. Foi um momento de intensa aprendizagem e de transformação de saberes e práticas, de acordo com os relatos. Notou-se também, lições afetivas, relacionados com o amor ao próximo e também com a humanização do cuidado. Destacam também a importância da prevenção, da organização e do planejamento das ações de saúde. A esse respeito, os profissionais de enfermagem relataram: 
A lição foi a humanização do cuidado, o amor ao próximo. (P2)

A lição foi prevenir sempre. (P4)

A lição foi que aprendi esse momento como de muita aprendizagem, de reavaliação de nossas condutas profissionais nos cuidados diários. (P5)

A lição foi que temos de estar sempre preparados, aos desafios que a profissão nos traz, buscando sempre novos conhecimentos, que não podemos parar. A enfermagem faz a diferença na vida das pessoas, que temos de atuar com amor, dedicação, mesmo diante das dificuldades e obstáculos, que surgem em nossa caminhada. (P9)

Acredito que tivemos duas principais lições: O trabalho em equipe é fundamental, pois cuidamos uns dos outros o tempo todo; e a fé deve fazer parte da nossa rotina. (P11)

A lição foi a importância da organização e planejamento das ações de saúde, de estarmos bem preparados e treinados para essa pandemia e as próximas que possam surgir, realizar treinamentos periódico para estar mais preparado. (P16)

Essa vivência, veio para nós mostrar, que um protocolo de trabalho bem estruturado, faz a diferença no atendimento ao paciente, que temos de dar mais valor as pequenas coisas e também as pessoas, o amor ao próximo. (P9)

Já a Classe $6(\mathrm{UCE}=15,4 \%)$ clarifica os impactos na vida pessoal em relação aos aspectos emocionais, familiares e sociais, sofridos pelos profissionais de enfermagem durante a pandemia de COVID-19. Os profissionais retratam os impactos sociais ocasionados sobretudo pelo isolamento e distanciamento social, evidenciado pela distância de seus familiares e entes queridos. Houve também, impactos de ordem financeira e de saúde, como o surgimento de transtornos psíquicos, como ansiedade, pânico e sintomas depressivos, conforme seguem os relatos:

Impactou na minha vida em todos os aspectos. Emocional pelas situações vivenciadas, familiar pois tenho filhos e não posso ficar muito próximo deles, o social foram as limitações. (P3)

Impactou na minha vida pessoal, na parte financeira e o distanciamento da família. (P4)

Impactou na minha vida pessoal, no emocional, em liderar outros profissionais com as mesmas inseguranças, com medo, e dúvidas constantes. (P5)

Impactou na minha vida pessoal, em muitos aspectos. Transtorno de ansiedade, pânico, tristeza por falta de socialização e dificuldade com as aulas on-line. (P7)

Resolvi sair de casa devido ao medo de contaminar minha família. Sobre a vida social permaneço em isolamento visto que tenho contato com casos confirmados diariamente. Com relação ao emocional já passei por vários sentimentos como medo, insegurança e ansiedade. (P11)

Impactou na minha vida pessoal, no convívio social sem dúvidas foi o mais impactante. Perdemos momentos que fazem a caminhada valer a pena. O mundo não será mais o mesmo depois dessa pandemia. (P12) 
A Classe 1 (UCE $=15,4 \%)$ demonstra as transformações ocorridas no processo de trabalho dos profissionais de enfermagem. Tais alterações dizem respeito ao foco na segurança dos usuários e profissionais, como o uso de paramentação adequada (EPIs), a mudança do fluxo de entrada nos serviços de saúde, uso de Veículos de Tráfego Rápido (VTR), criação de protocolos, planos de contingência e intensificação de normas de higiene, além de Unidades Básicas de Saúde (UBS) referências para o atendimento de casos suspeitos ou confirmados. Seguem as UCEs:

Mudou tudo, trabalho o tempo todo paramentada. Tenho que estar bem atenta aos sintomas do COVID-19, para que esse paciente seja isolado rapidamente e encaminhado para ala de suspeitos. (P13)

Mudou o fluxo de entrada, as paramentações com EPIs, a ambulância que foi montada especificamente para casos suspeitos e confirmados, e a proteção dos funcionários dentro do local de trabalho. (P15)

Os pacientes suspeitos ou com sintomas respiratórios, são direcionados para a Unidade Básica de Saúde de referência, realizamos a triagem e encaminhamos, evitando assim o contato do sintomático com outros pacientes não sintomáticos. (P9)

Atualmente temos veículo destinado somente para casos suspeitos ou confirmados, desinfecção dos materiais usados, maior proteção individual. (P4)

Centralização e especialização do atendimento ao paciente suspeito, treinamento constante da equipe de enfrentamento da Unidade Básica de Saúde, alinhamento municipal das notificações e fluxo de atendimento, várias outras situações conforme plano de contingência. (P5)

Planejo o local específico para atendimento dos casos suspeitos afastando do atendimento geral uso de máscaras e álcool gel, realizo orientação da equipe para como tem que ser o atendimento hoje independente de ser caso suspeito ou ñ̃o. (P16)

Mudou a gestão. Todo o processo de trabalho mudou e foi alterado, visto que neste momento a prioridade é garantir a segurança do atendimento e dos profissionais. (P11)

Por fim, a Classe $5(\mathrm{UCE}=15,4 \%)$ retrata o papel dos profissionais de enfermagem no enfrentamento da COVID-19. Segundo os mesmos, o papel deles é atuarem na prevenção, a partir de orientações de cuidados, bem como realizar encaminhamentos dos usuários aos serviços de referência, tratamento e reabilitação. Ainda, os enfermeiros atuam na coordenação das equipes de saúde, realizando o treinamento e capacitação dos demais profissionais, nos acompanhamentos de casos suspeitos e confirmados, e na elaboração de protocolos, conforme exposto nas seguintes UCEs:

O meu papel é o de prevenir, orientar, cuidar e encaminhar o paciente para o serviço necessário ao tratamento. Trabalho na linha de frente. (PI)

Estou na coordenação da equipe, meu papel é direcionar o trabalho de todos, treinar a equipe, orientar os cuidados. (P6)

Por trabalhar em uma Unidade Básica de Saúde, mesmo não sendo a referência para atendimento do COVID-19, temos de estar preparados a todo o momento para este tipo de atendimento. Também estar sempre informado sobre os assuntos, e as mudanças necessárias a este tipo de atendimento. (P9) 
Treinar as equipes assistenciais quanto as medidas de precauções para prevenir a contaminação dos profissionais $e$ pacientes.

Acompanhamento da evolução dos casos suspeitos e confirmados na minha área de abrangência e identificar novos suspeitos e orientar o seu direcionamento. (P8)

Elaborar ou aprovar junto as demais chefias protocolos que garantam a segurança em todos os processos (lavanderia, cozinha, assistência, descarte de resíduos). (P11)

Elaborar protocolo de paramentação e desparamentação. (P11)

\section{Discussão}

O enfrentamento da COVID-19 tem gerado sentimentos negativos nos profissionais de saúde em todo o mundo, segundo a classe 3, principalmente relacionados ao medo de auto contaminação, de infectar um membro da família ou da equipe (Asmundson \& Taylor, 2020). Tal contexto, pode impactar negativamente na saúde mental dos profissionais, os quais tem apresentado sintomas de estresse, depressão e ansiedade, inclusive alguns países orientais têm registrados maior número de casos de suicídios nessa população (Asmundson \& Taylor, 2020; Marins et al., 2020).

Como observado no presente estudo, na classe 6 , os profissionais de enfermagem destacaram alterações nos aspectos psicológicos, como desgaste emocional, o medo da contaminação e do novo, bem como aqueles relacionados com a dificuldade do processo de trabalho. Ainda, os impactos na vida pessoal fragilizam os aspectos emocionais, familiares e sociais, tornando os mesmos mais vulneráveis às alterações no bem estar (Chersich et al., 2020).

A manutenção de cuidados seguros e de qualidade na pandemia de COVID-19 depende da saúde e do bem-estar mental dos profissionais de saúde que atuam na linha de frente e também na gestão. A equipe de saúde enfrenta cansaço, difíceis decisões de triagem, separação de familiares e estigma, além da dor de perder pacientes e colegas, além de seus próprios riscos de infecção (Chersich et al., 2020).

Cuidar de pacientes infectados com a doença COVID-19 causa considerável estresse mental, resultando em altos níveis de ansiedade e transtornos de estresse pós-traumático, especialmente entre os enfermeiros (Huang, Han, Luo, Ren \& Zhou, 2020). Essas condições têm um grande impacto nos profissionais de saúde, mas também prejudicam sua capacidade de tomada de decisão e a qualidade da interação com os pacientes (Lijun et al., 2020). É importante ter em mente que a tensão experimentada no trabalho é agravada pelas mesmas rupturas e incertezas sentidas pelos membros da população em geral neste momento de pandemia (Catton, 2020).

Além disso, é importante salientar que a pandemia tem provocado uma crise social sem precedentes, fruto da alquimia das crises econômica, política e sanitária, acarretando graves consequências para a qualidade de vida e saúde da população menos favorecida (Fonseca, Fornari \& Lourenço, 2020).

Desse modo, os profissionais relataram, na classe 4, os desafios vivenciados pelos mesmos, como a falta de materiais, de Equipamento de Proteção Individual (EPIs), falta de protocolos, e a dificuldade em realizar atendimento de modo remoto. A experiência de trabalhar com Equipamentos de Proteção Individual (EPIs) limitados durante a pandemia também foi mencionada em um estudo realizado com enfermeiros nos Estados Unidos, em que observou-se a influência direta da falta de EPIs na saúde mental desses profissionais (Sousa et al., 2020).

Com isso, enfatiza-se a variedade de emoções vivenciadas, no qual incluem estarem assustados e com medo de ir para o trabalho, de contrair o vírus e trazê-lo para a família e amigos, medo do desconhecido, sensação de isolamento dos familiares, sentimento de raiva e traição do governo federal, falta de segurança para os profissionais, que se encontram exaustos pela 
demanda de trabalho, sentimento de luto, desamparo, perdidos e negação da realidade (Sousa, Santos, Silva \& Carvalho, 2020).

Observou-se também, classe 6, a preocupação destes profissionais com a segurança física pessoal, segurança psicológica, falta de segurança no emprego e segurança familiar, havendo aumento de sintomas relacionados a saúde mental devido o isolamento e aumento de violência doméstica.

O processo de trabalho da enfermagem usualmente já é estressante e permeado por sofrimento, com a pandemia tal contexto se agravou, o que compromete a saúde física e psíquica da categoria. Tal realidade demostra a necessidade urgente de implantação de medidas preventivas e de manejo dos fatores de risco com vistas a proteção da saúde dos trabalhadores da saúde em tempos de COVID-19 (Schultz et al., 2020).

É importante ressaltar que os serviços digitalizados ou de telemedicina podem reduzir o contato com o paciente e, portanto, os riscos de infecção. Assim, torna-se necessário que especialistas nacionais ou internacionais avaliem e aconselhem os profissionais de saúde remotamente. Embora possa haver custos consideráveis na instalação de sistemas para o desenvolvimento da telemedicina, eles podem ser compensados pela economia de Equipamentos de Proteção Individual (EPIs), recursos da equipe e melhores resultados para os pacientes. Essas iniciativas podem, no entanto, ser desafiadas por restrições de infraestrutura, como fornecimento de energia instável ou conectividade limitada com a internet e falta de interoperabilidade entre os sistemas digitais (Chersich et al., 2020).

Até o início de Dezembro de 2020, o Observatório da Enfermagem havia registrado 43.898 casos de profissionais da enfermagem infectado pelo novo coronavírus, e 466 mortes pela doença (COFEN, 2020). É necessário correlacionar os índices de contaminação e de óbitos, desses profissionais, com as condições de trabalho a que estão expostos, cotidianamente, no atendimento à população que busca assistência, vitimada ou com suspeita da COVID-19.

A mídia expõe a realidade do sistema de saúde: falta de EPIs, superlotação dos hospitais e das UPAs, equipes de saúde com baixas importantes de profissionais médicos, enfermeiros, técnicos/auxiliares de enfermagem e fisioterapeutas, devido às licenças médicas por adoecimento ou até mesmo por fazerem parte do grupo de risco e casos extremos, por óbito. Dada a emergência que impõe a pandemia, deve-se levar em conta também a possível ausência de práticas educativas para utilização do EPIs, bem como o manejo adequado dos mesmos com os pacientes infectados (Helioterio et al., 2020).

Em março de 2020, diante da necessidade de realizar o monitoramento remoto de indivíduos com COVID-19, o Conselho Federal de Enfermagem emitiu a Resolução COFEN no 634/2020 que autorizou e normatizou a teleconsulta de enfermagem como forma de combate à pandemia de COVID-19, mediante consultas, esclarecimentos, encaminhamentos e orientações com uso de meios tecnológicos.

$\mathrm{O}$ uso dos equipamentos e os norteamentos são essenciais, já que o papel dos profissionais de enfermagem no enfrentamento da COVID-19, de acordo com a classe 5, tem íntima relação com a execução do cuidado, manifestados desde ações de prevenção, a partir de orientações de cuidados, bem como na realização de encaminhamentos dos usuários aos serviços de referência, tratamento e reabilitação (Helioterio et al., 2020). Os enfermeiros atuam na coordenação das equipes de saúde realizando o treinamento e capacitação dos demais profissionais, no acompanhamento de casos suspeitos e confirmados, e na elaboração de protocolos. No âmbito da APS, especialmente, os enfermeiros acabam por realizar ambas ações: assistência e gestão.

Um problema sério ocorrido durante a pandemia tem sido o planejamento, preparação, organização e liderança inadequada de alguns governos e sistemas de saúde. Neste sentido, os enfermeiros necessitam de forte coragem moral e resiliência para prestar assistência. Estes profissionais precisam defender e estar envolvidos nas políticas para receber uma formação ética sólida para auxiliar na prestação de cuidados, bem como no gerenciamento de riscos durante emergências e desastres. Eles também precisam de liderança forte, direção clara e apoio contínuo uns dos outros, seus empregadores, o público e suas organizações de enfermagem para continuar a proteger as comunidades (Turale, Meechamnan \& Kunaviktikul, 2020). 
O enfrentamento da crise sanitária provocada pela COVID-19 no Brasil tem sido possível em razão do SUS contar com um enorme contingente de trabalhadores. A Enfermagem está presente em todas as etapas de nossas vidas e em todos os setores da saúde, desde a assistência ambulatorial ou hospitalar, na gestão pública do SUS em todas as esferas de governo, na educação, na pesquisa, na Ciência e Tecnologia, no controle social, entre outros, prestando serviços de alto valor social (Helioterio et al., 2020).

A reorganização advinda da pandemia, refletiu em transformações no processo de trabalho dos profissionais de enfermagem, tendo em vista a classe 1, especificamente no âmbito da segurança dos usuários e profissionais para a prevenção, com destaque para a paramentação adequada, higienização de ambientes, distanciamento, alterações nos fluxos e unidades de atendimento, desenvolvimento de protocolos e planos de contingência.

Um estudo realizado na África descreveu que uma gama de intervenções simples e de baixo custo podem reduzir a probabilidade de transmissão de infecção para profissionais de saúde em hospitais, incluindo máscaras para pacientes com sintomas respiratórios, lenços de papel para pacientes, promoção de etiqueta para tosse, lavagem das mãos e distanciamento social de pelo menos dois metros de distância. Porém, o distanciamento físico entre pacientes e pessoal administrativo, de limpeza e profissionais de saúde pode, no entanto, ser especialmente desafiador em unidades de saúde na APS superlotadas (Chersich et al., 2020).

A complexidade de ações para o enfrentamento da COVID-19 que podem ser sustentadas pela atuação da APS demanda que sejam priorizados três eixos de intervenção: o primeiro deles é fomentar ações de prevenção primária e secundária de vigilância em saúde com vistas a bloquear ou minimizar o aumento do número de casos no território; o segundo eixo consiste em oferecer suporte aos grupos com vulnerabilidades de saúde ou sociais que, sem dúvida, requererão algum tipo de apoio no transcorrer da epidemia; o terceiro eixo trata de garantir a continuidade das ações que eram desenvolvidas no contexto de promoção da saúde e prevenção de agravos no período anterior à pandemia da COVID-19 (ABRASCO, 2020).

Neste cenário, deve-se ter em mente que é preciso priorizar a continuidade do isolamento e do distanciamento social, quando possível. Assim, para reduzir índices de aglomeração e contatos presenciais desnecessários, abre-se a possibilidade de investir em novas ferramentas e estratégias do cuidado, como a promovida pelo contato telefônico de monitoramento no cenário da APS (ABRASCO, 2020).

Por fim, o processo de planejamento e atuação diante da pandemia rendeu lições para os profissionais de enfermagem, retratadas na classe 2, especialmente relacionadas com a aprendizagem e a transformação de saberes e práticas, não somente do ponto de vista de conhecimento científico voltados a prevenção, da organização e do planejamento das ações de saúde, mas também relacional e afetivos, relacionados com a empatia e humanização do cuidado.

Diante do cenário de catástrofe na saúde mundial, a enfermagem tem sido destaque. Em função da pandemia, está evidente a importância do trabalho da enfermagem e, mais que isso, a sociedade está valorizando e reconhecendo a profissão. A imprensa veiculando pesquisas desenvolvidas por enfermeiros, o trabalho humanizado nos serviços, o entendimento da complexidade da atuação da enfermagem, enfermeiros orientando a população sobre medidas preventivas, enfim, ficou evidente que o espaço que os trabalhadores de enfermagem ocupam é fundamental para o enfrentamento da COVID-19 e qualquer outra pandemia (Soares et al., 2020).

\section{Considerações Finais}

O estudo possibilitou compreender o processo de enfretamento da pandemia de COVID-19 na perspectiva de profissionais da enfermagem e de gestores municipais de saúde, e evidenciar que o enfrentamento não diferiu em relação ao nível de atenção em saúde que o profissional atua. As ações de saúde para a COVID-19 necessitaram de replanejamento e transformação no processo de trabalho da Enfermagem, e tal contexto epidemiológico gerou sentimentos negativos nos 
profissionais, conforme demonstrados na classe 3, como medo, insegurança, dúvidas e incertezas em relação ao contágio e transmissão do vírus, impactando diretamente na vida pessoal, familiar e social dos profissionais, com repercussões emocionas.

Assim, os principais desafios no enfrentamento da COVID-19 foram os aspectos psicológicos, aliados e até intensificados, pela dificuldade na execução do cuidado, com a falta de orientações e de conhecimentos sobre a doença, além de equipamentos de proteção adequados. Todo esse contexto destacou, na classe 5 , o papel da enfermagem na execução do cuidado e coordenação da equipe, fomentando a transformação de saberes e práticas, não somente em relação pandemia, mas também relacional.

Ressalta-se que o estudo foi realizado no início da pandemia, necessitando de novas pesquisas acerca da percepção da enfermagem no decorrer da mesma, bem como em momentos de novos picos de casos.

\section{Referências}

Araújo, J. L. D., Oliveira, K. K. D. D. \& Freitas, R. J. M. D. (2020). Em defesa do Sistema Único de Saúde no contexto da pandemia por SARS-CoV-2. Revista Brasileira de Enfermagem, 73(2), e20200247. http://dx.doi.org/10.1590/0034-7167-2020-0247

Asmundson, G. J. G. \& Taylor, S. (2020). Coronaphobia: Fear and the 2019-nCoV outbreak. Journal of Anxiety Disorders, 70, 102196. https://dx.doi.org/10.1016\%2Fj.janxdis.2020.102196

Associação Brasileira de Saúde Coletiva - ABRASCO. (2020). Desafios da APS no SUS no enfrentamento da Covid-19. Seminário Virtual Rede APS. Rede de Pesquisa em Atenção Primária à Saúde. http://www.abeno.org.br/arquivos/downloads/ABRASCO.pdf

Callaway, E. (2020). Time to use the p-word? Coronavirus enters dangerous new phase. Nature. https://www.nature.com/articles/d41586-020-00551-1

Catton, H. (2020). Global challenges in health and health care for nurses and midwives everywhere. International Nursing Review, 67(1), 4-6. https://doi.org/10.1111/inr.12578

Chersich, M. F., Gray, G., Fairlie, L., Eichbaum, Q., Mayhew, S., Allwood, B., English, R., Scorgie, F., Luchters, S., Simpson, G., Haghighi, M. M., Pham, M. D. \& Rees, H. (2020). COVID-19 in Africa: care and protection for frontline healthcare workers. Globalization and Heath, 16(46), 1-6. https://doi.org/10.1186/s12992-020-00574-3

Conselho Federal de Enfermagem - COFEN. (2020). Observatório da Enfermagem: Profissionais infectados com COVID-19 informado pelo serviço de saúde. http://observatoriodaenfermagem.cofen.gov.br/

Daumas, R. P., Silva, G. A. E., Tasca, R., Leite, I. D. C., Brasil, P., Greco, D. B., Grabois, V. \& Campos, G. W. D. S. (2020). O papel da atenção primária na rede de atenção à saúde no Brasil: limites e possibilidades no enfrentamento da COVID-19. Cadernos de Saúde Pública, 36, e00104120. http://dx.doi.org/10.1590/0102-311X00104120

Folha informativa COVID-19 - Escritório da OPAS e da OMS no Brasil. (2020a). Organização Pan-Americana da Saúde \& Organização Mundial da Saúde, OPAS/OMS. https://www.paho.org/pt/covid19

Fonseca, R. M. G. S. D., Fornari, L. F. \& Lourenço, R. G. (2020). Desafios da atenção básica no cuidado à população em tempo de pandemia. In: Organização Sheila Saint-Clair da Silva Teodósio \& Suderlan Sabino Leandro. Enfermagem na atenção básica no contexto da COVID-19. Brasília, DF: Associação Brasileira de Enfermagem/Departamento de Enfermagem na Atenção Básica (Série Enfermagem e Pandemias).

Helioterio, M. C., Lopes, F. Q. R. D. S., Sousa, C. C. D., Souza, F. D. O., Pinho, P. D. S., Sousa, F. N. E. F. D. \& Araújo, T. M. D. (2020). Covid-19: Por que a proteção de trabalhadores e trabalhadoras da saúde é prioritária no combate à pandemia?. Trabalho, Educação e Saúde, 18(3), e00289121. https://doi.org/10.1590/1981-7746-so100289

Huang, J., Han, M., Luo, T., Ren, A. \& Zhou, X. (2020). Mental health survey of medical staff in a tertiary infectious disease hospital for COVID-19. Chinese Journal of Occupational Health and Occupational Diseases, 38(0), e001. https://doi.org/10.3760/cma.j.cn121094-20200219-00063

Lana, R. M., Coelho, F. C., Gomes, M. F. D. C., Cruz, O. G., Bastos, L. S., Villela, D. A. M. \& Codeço, C. T. (2020). Emergência do novo coronavírus (SARSCoV-2) e o papel de uma vigilância nacional em saúde oportuna e efetiva. Cadernos de Saúde Pública, 36(3), e00019620. https://doi.org/10.1590/0102$311 \times 00019620$

Lijun, K., Yi, L., Shaohua, H, Min, C., Can, Y., Bing, X. Y., Ying, W., Jianbo, H., Jianbo, L., Xiancang, M., Jun, C., Lili, G., Gaohua, W., Hong, M. \& Zhongchun, L. (2020). The mental health of medical workers in Wuhan, China dealing with the 2019 novel coronavirus. The Lancet Psychiatry, 7(3), e14. https://doi.org/10.1016/S2215-0366(20)30047-X

Machado, M. H., Pereira, E. J., Neto, F. R. G. X. \& Wermelinger, M. C. D. M. W. (2020). Enfermagem em tempos da COVID-19 no Brasil: um olhar da gestão do trabalho. Enfermagem em Foco, 11(1) Especial, 32-39. https://doi.org/10.21675/2357-707X.2020.v11.n1.ESP.3994

Marins, T. V. D. O., Crispim, C. G., Evangelista, D. D. S., Neves, K. D. C., Fassarella, B. P. A., Ribeiro, W. A. \& Silva, A. A. D. (2020). Enfermeiro na linha de frente ao COVID-19: A experiência da realidade vivenciada. Research, Society and Development, 9(8), e710986471. https://doi.org/10.33448/rsd-v9i8.6471 
Research, Society and Development, v. 10, n. 1, e5410111252, 2021

(CC BY 4.0) | ISSN 2525-3409 | DOI: http://dx.doi.org/10.33448/rsd-v10i1.11252

Ministério da Saúde (2020c). Plano de Contingência Nacional para Infecção Humana pelo novo Coronavírus COVID-19. Secretaria de Vigilância em Saúde. Brasília, DF: Ministério da Saúde.

Schultz, C. C., Corrêa, K. I. D., Vaz, S. M. C., Colet, C. D. F. \& Stumm, E. M. F. (2020). Resiliência da equipe de enfermagem no âmbito hospitalar com ênfase na pandemia COVID-19. Research, Society and Development, 9(11), e539119466. http://dx.doi.org/10.33448/rsd-v9i11.9466

Soares, S. S. S., Souza, N. V. D. D. O., Carvalho, E. C., Varella, T. C. M. Y. M. L., Andrade, K. B. S. D., Pereira, S. R. M. \& Costa, C. C. P. D. (2020). De cuidador a paciente: na pandemia da Covid-19, quem defende e cuida da enfermagem brasileira?. Escola Anna Nery, 24(spe), e20200161. http://dx.doi.org/10.1590/2177-9465-ean-2020-0161

Sobre a doença: O que é COVID-19 (2020d). Ministério da Saúde. https://coronavirus.saude.gov.br/sobre-a-doenca\#transmissao

Sousa, A. R. D., Santos, G. L. A., Silva, R. S. D. S. \& Carvalho, E. S. D. S. (2020). Reflexões sobre o Processo de Enfermagem no trabalho de enfermeiras frente à pandemia da Covid-19. Enfermagem em Foco, 11(1) Especial, 62-67. https://doi.org/10.21675/2357-707X.2020.v11.n1.ESP.3501

Turale, S., Meechamnan, C. \& Kunaviktikul, W. (2020). Challenging times: ethics, nursing and the COVID-19 pandemic. International Nursing Review, 67(2), 164-167. https://doi.org/10.1111/inr.12598 